\title{
Interaction between Bordetella bronchiseptica and Toxigenic Pasteurella multocida on the Nasal Mucosa of SPF Piglets
}

\author{
Béla ÉLIÁS, Mihály ALBERT ${ }^{1)}$, Sándor TUBOLY'), and Pál RAFAI \\ Department of Animal Hygiene, ${ }^{1)}$ Pathological Anatomy and ${ }^{2)}$ Epizootiology, University of Veterinary Science, H-1400 Budapest, Pf. 2 , \\ Hungary
}

(Received 31 October 1991/Accepted 30 July 1992)

\begin{abstract}
The interaction between Bordetella bronchiseptica and type D toxigenic Pasteurella multocida was studied in five groups of 4 specific-pathogen-free (SPF) piglets each. At 28 days of age, piglets of groups 3 and 4 were inoculated into both nostrils with $10^{8}$ colony-forming-units (CFU) of a non-dermonecrotic toxin (DNT)-producing, phase I strain of $B$. bronchiseptica. Piglets of groups 1 and 3 were treated intranasally with a sonic extract of the non-toxic strain of $B$. bronchiseptica and those of groups 2 and 4 with $B$. bronchiseptica DNT into the left nostril. Sonic extract and DNT treatment was started at 33 days of age and lasted for 5 days. Piglets of group 5 served as controls. At the age of 37 days, piglets of all groups except group 5 were inoculated into both nostrils with $5 \times 10^{7} \mathrm{CFU}$ of toxigenic $P$. multocida. At slaughter at 50 days of age, $P$. multocida was recovered from the left nasal cavity of 3 piglets of group 2 and all piglets of group 4. In piglets inoculated with $B$. bronchiseptica DNT the mucosal epithelial cells of the left nasal cavity showed loss of cilia, regressive lesions such as vacuolation, karyopycnosis and necrosis, hypertrophy of the epithelium, infiltration of the epithelium and submucosa by inflammatory cells, could also be seen. The results suggest that action of the $B$. bronchiseptica DNT on the nasal mucosa is a precondition of the growth of $P$. multocida in the nasal cavity. It appears that colonization by $P$. multocida presupposes presence of a mucosal injury inflicted by $B$. bronchiseptica $D$ DT in itself, without involvement of the other virulence factors.-KEY wonDs: atrophic rhinitis, Bordetella bronchiseptica, dermonecrotic toxin (DNT), interaction, Pasteurella multocida.
\end{abstract}

J. Vet. Med. Sci. 54(6): 1105-1110, 1992

The combination of toxigenic Bordetella bronchiseptica $[13,15]$ and toxigenic Pasteurella multoci$d a[17,21]$ in causing atrophic rhinitis (AR) in swine is well known. The importance of $P$. multocida became clear when its toxigenic strains were discovered [9] and found to induce progressive AR following experimental infection $[16,20]$. Gnotobiotic piglets experimentally infected with toxigenic $B$. bronchiseptica sometimes developed lesions with a tendency to heal [19]. Combined infection of the experimental piglets nasal cavity with both toxigenic bacteria led to severe atrophy of the nasal turbinates [20]. In the absence of $B$. bronchiseptica, $P$. multocida cannot grow on the healthy nasal mucosa $[4,17]$. After experimental infection of gnotobiotic piglets only a small number of $P$. multocida colonized on the nasal mucosa and produced only mild atrophy [20], confirming that the presence of $B$. bronchiseptica is important for the growth of $P$. multocida in the nasal cavity. In the absence of $B$. bronchiseptica, colonization of the porcine nasal mucosa by toxigenic $P$. multocida could be enhanced by intranasal inoculation of $1 \%$ acetic acid before infection [7]. Chanter et al. [2] suggested that, besides the $B$. bronchiseptica toxin, other products of $B$. bronchiseptica and the interaction between the two species may be important in colonization of the nasal mucosa by $P$. multocida . However, the role of the virulence factors of $B$. bronchiseptica (adhesins, DNT, adenylate cyclase) in this process was not studied in detail previously.

The aims of the experiments described here were to determine whether the simultaneous presence of $B$. bronchiseptica is needed for colonization of the porcine nasal mucosa by toxigenic $P$. multocida, and to characterize the potential interaction, if any, between the two bacteria.

\section{MATERIALS AND METHODS}

Bacterial strains: B. bronchiseptica strain 219 was isolated from pig affected with clinical AR [5]. $B$. bronchiseptica strain N-95 was isolated from the nasal cavity of a pig free from the clinical signs and gross pathological lesions of AR at slaughter. Both strains showed phase I colony morphology and were hemolytic on Bordet-Gengou (BG) agar (Difco, U.S.A.) containing $10 \%$ bovine blood [6]. They produced adenylate cyclase and hemagglutinin for horse, calf and sheep (titres of strain 219: 1:16, 1:16 
and 1:32; titres of strain N-95:1:16, 1:32 and 1:8, respectively). Both strains possessed all $\mathrm{K}$ antigenic determinants except factor 13. Strain 219 also produced mouse lethal factor and dermonecrotic toxin in guinea pigs (necrosis $19 \mathrm{~mm}$ in diameter), whereas strain N-95 did not [6].

P. multocida strain 9591 was isolated from the nasal cavity of a piglet affected with AR. The strain belonged to type $\mathrm{D}$ and produced dermonecrotic toxin (necrosis $24 \mathrm{~mm}$ in diameter) [5].

Purification and testing of dermonecrotic toxin $(D N T)$ : Preparation of DNT from toxigenic $B$. bronchiseptica strain 219 and determination of its activity were carried out by the methods described previously [5]. The preparation was checked for purity by two-dimensional double gel diffusion [5] and by PAGE. To rule out any nonspecific activity, the concentrated DNT was dialysed before use. DNT gave a single precipitation line by gel diffusion and a double band by PAGE using Coomassie blue staining. Its biological activity was ca. $150 \mathrm{ng}$ in the guinea pig skin test and its $\mathrm{LD}_{50}$ to mice was ca. 500 ng.

Preparation of non-toxic extract: The bacterial extract was prepared from a non-toxic suspension of B. bronchiseptica strain N-95 containing $10^{12} \mathrm{CFU} /$ $\mathrm{m} l$ by sonication at $50 \mathrm{~Hz}$ for $10 \mathrm{~min}$, then filtered through a $0.22 \mu \mathrm{m}$ Millipore filter.

Inocula for piglets: $B$. bronchiseptica inoculum was prepared from $B$. bronchiseptica strain N-95. The isolate was cultured on BG agar containing $10 \%$ bovine blood at $37^{\circ} \mathrm{C}$ for $24 \mathrm{hr}$. The inoculum was prepared with phosphate buffer of $\mathrm{pH} 7.2$ and adjusted to a concentration of $2 \times 10^{8}$ colonyforming-units (CFU)/ml by spectrophotometry (SPECOL-10, Karl Zeiss Jena, Germany). P. multocida inoculurn was prepared by the method of Éliás et al. [4]. The inoculum was adjusted to $10^{8}$ $\mathrm{CFU} / \mathrm{m} l$.

Experimental infection in SPF piglets: Four-weekold SPF piglets were assigned to 5 groups of 4 piglets each and kept isolated in climatic chambers up to 50 days of age when they were killed.

The piglets were obtained from hysterectomyderived sows free from $B$. bronchiseptica, $P$. multocida, Mycoplasma hyopneumoniae, Actinobacillus pleuropneumoniae, Haemophilus parasuis, and from antibodies to these microorganisms.

From the age of 33 days on, piglets of group 1 were inoculated into the left nostril with a non-toxic bacterial extract containing $2 \mu \mathrm{g}$ protein. The inoculum was given 5 times daily, on 5 consecutive days. Piglets of group 2 received, into the left nostril, $2 \mu \mathrm{g}(0.5 \mathrm{~m} l) B$. bronchiseptica DNT 5 times daily, on 5 consecutive days, starting when the piglets were 33 days old.

At the age of 28 days, piglets of group 3 were inoculated with $B$. bronchiseptica $\mathrm{N}-95$ into both nostrils. The inoculum contained $10^{8} \mathrm{CFU} / 0.5 \mathrm{~m} l$ per nostril. From the age of 33 days, the piglets received, into the left nostril, $B$. bronchiseptica N-95 bacterial extract containing $2 \mu \mathrm{g}$ protein 5 times daily, on 5 consecutive days.

When 28 day-old, piglets of group 4 were inoculated with $B$. bronchiseptica $\mathrm{N}-95$ into both nostrils. The inoculum contained $10^{8} \mathrm{CFU} / 0.5 \mathrm{~m} l$ per nostril. From the age of 33 days, the piglets received, into the left nostril, $2 \mu \mathrm{g} B$. bronchiseptica DNT 5 times daily, on 5 consecutive days.

At the age of 37 days, piglets of all groups except group 5 were inoculated, into both nostrils, with toxigenic, type D P. multocida $\left(5 \times 10^{7} \mathrm{CFU} / 0.5 \mathrm{ml}\right.$ per nostril).

Piglets of group 5 served as untreated controls.

Bacteriological examination: Nasal swab samples for bacteriological examination were taken from the piglets at 5-day intervals. At slaughter, swabs from the surface of the pharyngeal tonsils were also cultured for bacteria. B. bronchiseptica was isolated from nasal swabs after plating on BG agar plates containing $10 \%$ bovine blood. Plates were incubated at $37^{\circ} \mathrm{C}$ for $24 \mathrm{hr}$. P. multocida was isolated on YPC agar (cystine agar medium containing yeast- proteose peptone, Difco) containing 10\% sterile bovine blood after incubation at $37^{\circ} \mathrm{C}$ for $18 \mathrm{hr}$ [14].

The strains were identified by the criteria of Bergey's Manual [18, 22]. Phase type, capsular antigens, haemagglutinin of $B$. bronchiseptica were determined as described by Éliás and Kruger [6] and adenylate cyclase activity was tested by the method of Kruger et al. [11]. The capsular antigens of $P$. multocida strains were determined by indirecthaemagglutination and by the acriflavine and hyaluronidase tests [1].

B. bronchiseptica DNT activity was determined by the skin test in guinea pigs and by a mouse test. Albino guinea pigs weighing $500 \mathrm{~g}$ were inoculated intracutaneously with $0.2 \mathrm{~m} l$ of the DNT. The minimal dose of DNT was the highest dilution of the DNT which caused cutaneous necrosis $10 \mathrm{~mm}$ in diameter in $72 \mathrm{hr}$. To determine the $\mathrm{LD}_{50}$ of the DNT, four SPF mice weighing $20 \mathrm{~g}$ were inoculated 
Table 1. The effect of various pretreatments of pigs with Bordetella bronchiseptica extract or cells on the subsequent colonization of the nasal mucosa by Pasteurella multocida

\begin{tabular}{|c|c|c|c|c|c|c|c|c|c|c|}
\hline \multirow{4}{*}{$\begin{array}{l}\text { Group } \\
(n=4)\end{array}$} & \multicolumn{3}{|c|}{ Pretreatment } & Challenge & \multicolumn{6}{|c|}{ Recovery } \\
\hline & \multicolumn{3}{|c|}{ B. bronchiseptica } & P. multocida & \multicolumn{3}{|c|}{ B. bronchiseptica } & \multicolumn{3}{|c|}{ P. multocida } \\
\hline & \multirow{2}{*}{$\begin{array}{c}\text { Live } \\
\text { culturea) } \\
\text { (non- } \\
\text { toxigenic) }\end{array}$} & \multirow[t]{2}{*}{$\begin{array}{l}\text { Non-toxic } \\
\text { extract }^{\text {) }}\end{array}$} & \multirow[t]{2}{*}{$\mathrm{DNT}^{\mathrm{c})}$} & \multirow[t]{2}{*}{$\begin{array}{l}\text { Live culture }{ }^{\mathrm{d})} \\
\quad \text { (toxigenic) }\end{array}$} & \multicolumn{2}{|c|}{$\begin{array}{l}\text { Nasal } \\
\text { cavity }\end{array}$} & \multirow[t]{2}{*}{ Tonsils } & \multicolumn{2}{|c|}{$\begin{array}{l}\text { Nasal } \\
\text { cavity }\end{array}$} & \multirow[t]{2}{*}{ Tonsils } \\
\hline & & & & & Right & Left & & Right & Left & \\
\hline 1 & - & + & - & + & $0 / 4^{\mathrm{e})}$ & $0 / 4$ & $0 / 4$ & $0 / 4$ & $0 / 4$ & $0 / 4$ \\
\hline 2 & - & - & + & + & $0 / 4$ & $0 / 4$ & $0 / 4$ & $0 / 4$ & $3 / 4$ & $4 / 4$ \\
\hline 3 & + & + & - & + & $4 / 4$ & $4 / 4$ & $0 / 4$ & $0 / 4$ & $0 / 4$ & $0 / 4$ \\
\hline 4 & + & - & + & + & $4 / 4$ & $4 / 4$ & $0 / 4$ & $0 / 4$ & $4 / 4$ & $4 / 4$ \\
\hline 5 & - & - & - & - & $0 / 4$ & $0 / 4$ & $0 / 4$ & $0 / 4$ & $0 / 4$ & $0 / 4$ \\
\hline
\end{tabular}

a) Inoculated into both nostrils at 28 days of age.

b) Treated into the left nostril on 5 consecutive days, from 33 to 37 days of age.

c) Treated as above (b).

d) Inoculated into both nostrils at 37 days of age.

e) Infected piglets/tested.

See text for further details.

intraperitoneally with $0.2 \mathrm{~m} l$ of the serial dilution of each DNT. The test was read $72 \mathrm{hr}$ after inoculation $[5]$.

Pathological and histological examination: The head of the piglets was split longitudinally and examined for lesions of the nasal turbinates, nasal septum and ethmoid bone.

Tissue specimens secured for histopathological examination, transmission electron microscopy (TEM) and scanning electron microscopy (SEM) were treated and examined by the method described previously [5].

\section{RESULTS}

Bacteriological findings: The nasal swabs of group $I$ and group 5 piglets were free from $B$. bronchisepti$c a$ and $P$. multocida throughout.

The left nasal cavity of 3 and tonsil of 4 group 2 piglets yielded $P$. multocida at 40 and 45 day-old and at slaughter at the age of 50 days.

B. bronchiseptica strain N-95 was consistently cultured from both the right and the left nasal cavities of group 3 piglets beyond the age of 30 days. $P$. multocida, however, was not isolated from these piglets.

B. bronchiseptica strain N-95 was consistently isolated from both the right and the left nasal cavities of group 4 piglets on every 5 th day after 30 days of age. In the period between 40 days of age and slaughter, the left nasal cavity and tonsil of 4 piglets yielded $P$. multocida (Table 1).
B. bronchiseptica and $P$. multocida strains isolated from the experimental piglets had the same properties as those contained in the inocula.

Histopathological findings: Piglets of all groups were free from gross lesions in the nasal cavity at slaughter. Neither light microscopic nor electron microscopic lesions were demonstrable in either of the nasal turbinates of piglets of groups 1,3 and 5 and in the right nasal cavities of group 2 and 4 piglets. In piglets intranasally treated with $B$. bronchiseptica DNT the mucosal epithelial cells of the left turbinate showed loss of cilia and of microvilli (Fig. 1). Regressive lesions such as vacuolation, karyopycnosis and necrosis, hypertrophy of the epithelium, widening of the intracellular spaces, and infiltration of the epithelium and submucosa by inflammatory cells such as lymphocytes, plasma cells and granulocytes could also be seen (Fig. 2).

\section{DISCUSSION}

The poor ability of $P$. multocida to colonize on the porcine nasal mucosa [12] inspired investigations into the factors promoting colonization in given conditions. Studies along this line demonstrated the favorable influence of mucosal infection by Haemophilus parasuis [8] and of painting with $1 \%$ acetic acid [7] in experimental conditions. Pedersen and Barfod [17] studied the influence of noninfectious factors in natural $P$. multocida infections in which isolation experiments failed to detect the 


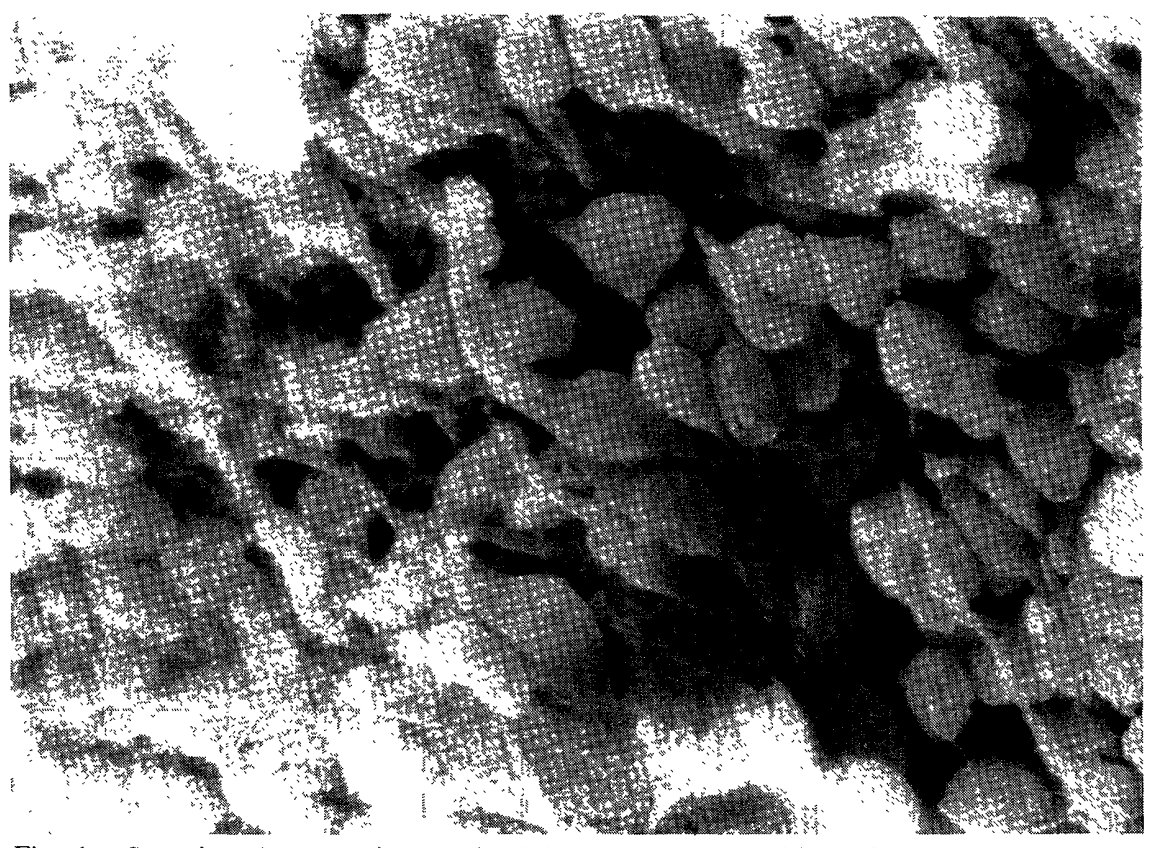

Fig. 1. Scanning electron micrograph of the ventral nasal turbinate from a 50-day-old SPF piglet intranasally treated with $10 \mu \mathrm{g}$ of Bordetella bronchiseptica DNT five times. The mucosal epithelial cells of the turbinate show loss of cilia and microvilli. $\times 7,500$.

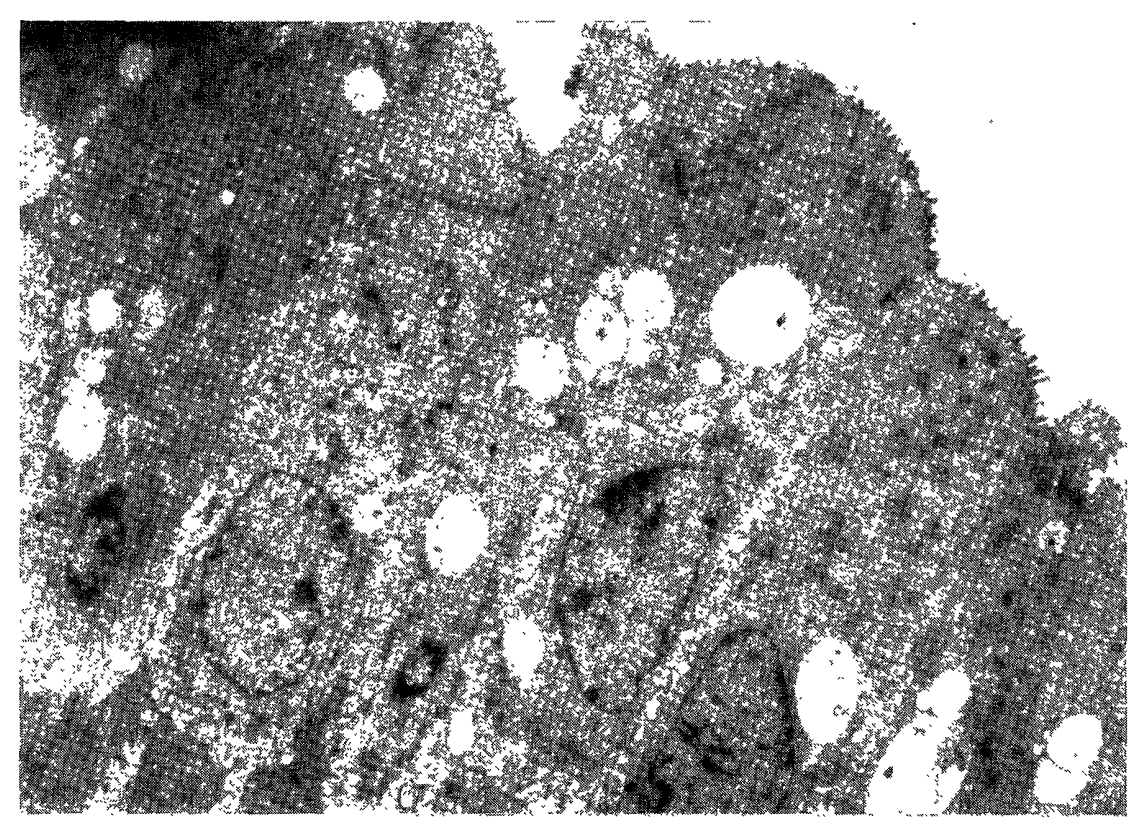

Fig. 2. Transmission electron micrograph of the ventral nasal turbinate from a 50 -day-old SPF piglet intranasally treated with $10 \mu \mathrm{g}$ of Bordetella bronchiseptica DNT five times. The nasal mucosal epithelial cells show regressive lesions such as vacuolation, karyopycnosis and necrosis. Widenings of the intracellular spaces are seen. $\times 5,000$.

simultaneous presence of $B$. bronchiseptica. Chanter and Rutter [3] reported that the extent of a $P$. multocida infection induced in acid-painted nasal mucosa could be reduced by simultaneous administration of $P$. multocida antitoxin. This supports the conclusion that the DNT produced by $P$. multocida promoted colonization of the nasal mucosa by the DNT-producing strain.

In addition, authors generally agree that the presence of toxin-producing $B$. bronchiseptica 
promotes the establishment and growth of $P$. multocida on the nasal mucosa [20].

In view of this, we investigated the influence of $B$. bronchiseptica DNT in itself, and the joint influence of non-toxic factors, on colonization of the nasal mucosa by $P$. multocida. Four experimental groups were formed to study the effect of a non-toxic $B$. bronchiseptica extract (group 1), a non-DNTproducing live B. bronchiseptica strain (group 3) and of $B$. bronchiseptica DNT alone (group 2) and in combination (group 4).

Treatment with the non-toxic $B$. bronchiseptica extract (group 1) failed to promote the growth of $P$. multocida and infection with a non-DNT-producing $B$. bronchiseptica strain (group 3) had no influence either on colonization by $P$. multocida, unlike the pertinent observations of Chanter et al. [2]. The inconsistent results may have been due to the fact that not only the initial age of the experimental piglets, but also the period of study, the method of bacterial isolation and the properties of the nonDNT-producing B. bronchiseptica strains used for experimental infection differed between the experiments performed by Chanter et al. [2] and ourselves.

We isolated $P$. multocida colonies from the nasal mucosa of the piglets intranasally treated with $B$. bronchiseptica DNT. It follows that the mucosal injury inflicted by DNT was sufficient in itself, without a simultaneous presence of $B$. bronchiseptica organisms, to promote colonization by $P$. multocida. It also follows that other virulence factors, such as adenylate cyclase and adhesins, played no role whatever in the process studied.

The positive response of the piglets infected with non-DNT-producing $B$. bronchiseptica and treated additionally with $B$. bronchiseptica DNT by the intranasal route (group 4) can be explained as follows: P. multocida may have "realized" the fact of being "cheated" by us if its colonization had in fact depended on interaction between the two bacteria. In that case no colonization would have taken place. Since, however, the $P$. multocida organisms did grow to the same extent as in the nasal mucosa of the piglets of group 2, the results obtained in group 4 unequivocally supported the independent action of DNT.

The bacteriological and pathological results obtained for the nasal cavities of group 2 and group 4 piglets give answers to further two important questions. Firstly, it has become obvious that $P$. multocida can colonize and grow only on a nasal mucosa the epithelial cells of which show regressive changes detectable at least by microscopy. This statement is supported by the finding that $P$. multocida grew only in the piglets left nasal cavity showing pathological changes, and could not be recovered throughout the experiment from the right nasal cavity having an intact mucous membrane. Secondly, it has been revealed that in piglets older than 33 days a 5-day treatment of the nasal mucosa with $B$. bronchiseptica DNT and a toxigenic $P$. multocida infection existing for 13 days are not sufficient to produce gross lesions of the nasal bones.

The present experimental observations pose the question whether the action of $B$. bronchiseptica DNT is a specific prerequisite of the growth of $P$. multocida, or just represents an effect that may as well be exerted by other factors promoting colonization. The clarification of this problem would be of great practical importance, despite the fact that $B$. bronchiseptica infection is omnipresent in conventional pig herds all over the world $[10,20]$.

ACKNOWLEDGEMENT. We thank the National Research Fund (OTKA) for financial support which enabled us to carry out this work.

REFERENCES

1. Carter, G. R. and Subronto, P. 1973. Identification of type $\mathrm{D}$ strains of Pasteurella multocida with acriflavine. Am. J. Vet. Res. 34: 293-294.

2. Chanter, N., Magyar, T., and Rutter, J. M. 1989. Interaction between Bordetella bronchiseptica and toxigenic Pasteurella multocida in atrophic rhinitis of pigs. Res. Vet. Sci. 47: 48-53.

3. Chanter, N. and Rutter, J. M. 1990. Colonisation by Pasteurella multocida in atrophic rhinitis of pigs and immunity to the osteolytic toxin. Vet. Microbiol. 25: 253-265.

4. Éliás, B., Herpay, Mária, Albert, M., Fekete Judit, Tuboly, S., Rafai, P., and Molnár, Edit 1990a. The influence of growth conditions of Pasteurella multocida on its ability to colonise the nasal mucosa of SPF piglets. Vet. Microbiol. 24: 81-88.

5. Éliás, B., Boros, G., Albert, M., Tuboly, S., Gergely, P., Papp, L., Barna Vetro, Ildikó, Rafai, P., and Molnár, Edit 1990b. Clinical and pathological effects of the dermonecrotic toxin of Bordetella bronchiseptica and Pasteurella multocida in specific-pathogen-free piglets. Jpn. J. Vet. Sci. 52: $677-688$.

6. Éliás, B. and Krüger, Monika 1988. Untersuchungen zur Differenzierung von Bordetella bronchiseptica-Stämmen. 5. Mitteilung: Virulenzfaktoren in Beziehung zum Phasenwechsel bei Bordetella bronchiseptica-Stämmen. Arch. Exp. Vet. Med. 42: 859-866. 
7. Elling, F. and Pedersen, K. B. 1985. The pathogenesis of persistent turbinate atrophy induced by toxigenic Pasteurella multocida in pigs. Vet. Pathol. 22: 469-474.

8. Gois, M., Barnes, H. J., and Ross, R. F. 1983. Potentiation of turbinate atrophy in pigs by long-term nasal colonisation with Pasteurella multocida. Am. J. Vet. Res. 44: 372-378.

9. Ilina, Z. M. and Zasukhin, M. I. 1975. Role of Pasteurella toxins in the pathogenesis of infectious atrophic rhinitis. Sbornik Nauch. Rabot Siberskii Nauch, Issled. Vet. Inst. Omsk 25: 76-80.

10. Kielstein, P. and Éliás, B. 1985. Zur Bedeutung von Bordetella bronchiseptica und Pasteurella multocida bei der Rhinitis atrophicans Suum. Zentralbl. Veterinaermed., B 32: 694-705.

11. Krüger, Monika, Lux, R., Schilow, W., and Horsch, F. 1988. Untersuchungen zur Differenzierung von Bordetella bronchiseptica-Stämmen. 7. Mitteilung: Phänotypische Veränderungen bei Bordetella bronchiseptica durch Phasenwechsel. Arch. Exp. Vet. Med. 42: 213-220.

12. Kume, K., Nakai, T., Yoshikawa, H., Oyamada, T., and Yoshikawa, T. 1986. Adherence of Pasteurella multocida and Bordetella bronchiseptica to the swine nasal epithelial cells and possible role in virulence. Proc. 9th Int. Pig Vet. Soc. Congr. Barcelona (Spain), p. 233.

13. Nakai, T., Kume, K., Yoshikawa, H., Oyamada, T., and Yoshikawa, T. 1985. Changes in the nasal mucosa of specific-pathogen-free neonatal pigs infected with Pasteurella multocida or Bordetella bronchiseptica. Jpn. J. Vet. Sci. 48: 693-701.

14. Namioka, S. and Murata, M. 1961. Serological studies on Pasteurella multocida. 1. A simplified method for capsular typing of the organism. Cornell Vet. 51: 498-507.

15. Oyamada, T., Yoshikawa, T., Yoshikawa, H., Shimizu, M., Nakai, T., and Kume, K. 1986. Lesions induced in the nasal turbinates of neonatal pigs by Pasteurella multocida or Bordetella bronchiseptica. Jpn. J. Vet. Sci. 48: 377-387.

16. Pedersen, K. B. and Barfod, K. 1981. The aetiological significance of Bordetella bronchiseptica and Pasteurella multocida in atrophic rhinitis of swine. Nord. Vet. Med. 33: 513-522.

17. Pedersen, K. B. and Barfod, K. 1982. Effect of the incidence of atrophic rhinitis on vaccination of sows with a vaccine containing Pasteurella multocida toxin. Nord. Vet. Med. 34: 293-302.

18. Pittmann, M. 1974. Genus Bordetella Moreno-Lopes. pp. 282-283. In: Bergey's Manual of Determinative Bacteriology, 8th ed. (Buchanan, R. E. and Gibbons, N. E. eds.), Williams and Wilkins, Baltimore.

19. Rutter, J. M. 1981. Quantitative observations on Bordetella bronchiseptica infection in atrophic rhinitis of pigs. Vet. Rec. 108: 451-454.

20. Rutter, J. M. and Rojas, X. 1982. Atrophic rhinitis in gnotobiotic piglets: differences in the pathogenicity of Pasteurella multocida in combined infections with Bordetella bronchiseptica. Vet. Rec. 110: 531-535.

21. Schöss, P. 1989. Die Rhinitis atrophicans des Schweineseine Faktorenkrankheit? Berl. Münch. Tierärztl. Wochenschr. 102: 387-389.

22. Smith, J. E. 1974. Genus Pasteurella. pp. 370-373. In: Bergey's Manual of Determinative Bacteriology, 8th ed. (Buchanan, R. E. and Gibbons, N. E. eds.), Williams and Wilkins, Baltimore. 